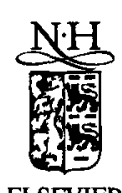

ELSEVIER

Nuclear Physics B 500 (1997) 405-420

\title{
Simplifying the spectral analysis of the volume operator
}

\author{
R. Loll ${ }^{1}$ \\ Max-Planck-Institut für Gravitationsphysik, Schlaatzweg 1, D-14473 Potsdam, Germany \\ Received 17 March 1997; accepted 21 May 1997
}

\begin{abstract}
The volume operator plays a central role in both the kinematics and dynamics of canonical approaches to quantum gravity which are based on algebras of generalized Wilson loops. We introduce a method for simplifying its spectral analysis, for quantum states that can be realized on a cubic three-dimensional lattice. This involves a decomposition of Hilbert space into sectors transforming according to the irreducible representations of a subgroup of the cubic group. As an application, we determine the complete spectrum for a class of states with six-valent intersections. (C) 1997 Elsevier Science B.V.
\end{abstract}

PACS: 04.60.Ds; 04.60.Nc; 02.20.Rt

Keywords: Canonical quantum gravity; Volume operator; Lattice gravity; Determinant of the metric; Octagonal group

\section{Introduction}

Researchers in gravitational physics these days can look back on a ten-year long effort of quantizing the theory canonically in terms of a set of "new" connection variables [1]. These developments have led to many new insights, but at the same time have not been free of Irrungen und Wirrungen, and the process is by no means finished yet.

From a technical point of view, the problem of representing non-polynomial quantities in the quantum theory (a central difficulty in ADM-type quantization approaches) in this formulation has now been recast into that of diagonalizing the operator $\operatorname{det} E$, where $\operatorname{det} E$ is up to a sign the classical determinant $\operatorname{det} g$ of the Riemannian metric $g_{a b}$ on spatial

\footnotetext{
'E-mail: loll@aei-potsdam.mpg.de
} 
slices. The fact that non-polynomiality can be re-expressed as "polynomiality modulo powers of $\sqrt{\operatorname{det} g}$ " is not specific to the connection formulation (see, for example, Ref. [2]). However, what distinguishes this new approach is the fact that (certain functions of) det $g$ have well-defined finite, self-adjoint operator analogues.

The reason for this is that in the quantization the classical conjugate variable pairs $\left(A_{a}^{i}, E_{i}^{a}\right)$ - like in Yang-Mills theory - are represented by multiplication and differentiation with respect to $A_{a}^{i}$. This implies that suitably quantized versions of the classical determinant

$$
\operatorname{det} E(x)=\frac{1}{3 !} \eta_{a b c} \epsilon^{i j k} E_{i}^{a}(x) E_{j}^{b}(x) E_{k}^{c}(x)
$$

are third-order differential operators. Moreover, if the quantization is based on onedimensional flux line states, as is the case in the so-called "loop quantization" schemes [3,4], and also in lattice-discretized versions of canonical quantum gravity [5], $E$-flux is quantized. This is ultimately responsible for the finiteness of quantities like the volume operator, which is the quantization of the integral $\int_{\mathcal{R}} d^{3} x \sqrt{\operatorname{det} E}$, for a spatial region $\mathcal{R}$.

The framework we will be using in the following is not the one originally proposed in [1], since the reality conditions that have to be imposed on its $s l(2, \mathbb{C})$-valued connections $A_{a}^{i}(x)$ seem to be intractable in the quantum theory. Instead, we will use a closely related version in terms of real, $s u(2)$-valued connection forms [6], which avoids this difficulty. In this formulation, the Hamiltonian constraint function is nonpolynomial.

The subject of this paper is the introduction of a method for simplifying the diagonalization of the volume operator (and potentially other operators relevant in loop quantum gravity), by exploiting symmetries of the Wilson loop states that form the Hilbert space it is defined on. Our discussion will take place in the discretized version of the theory on a cubic lattice. The discrete volume operator one can define on the lattice $[7,8]$ is closely related to the finite volume operators one obtains after regularization in the continuum theory [9-14]. The type of intersection of Wilson loop states that can occur on the lattice is at most six-valent, and therefore not the most general from the point of view of the continuum theory, but also in this case it may well be sufficiently generic from a physical standpoint.

The operator $\widehat{\operatorname{det} E}$ occurs in a variety of contexts. It was originally conceived as an ingredient in the definition of the volume operator, i.e. the quantization of $\int_{\mathcal{R}} d^{3} x \sqrt{\operatorname{det} E}$ mentioned above $[9,10]$. Defined on the kinematical Hilbert space of the loop representation, this kind of geometric operator enables one to associate well-known geometric properties with Wilson loop states, or possibly coarse-grained ensembles of such states [9].

With the return to real connection variables in canonical quantum gravity, knowledge of the spectrum of $\widehat{\operatorname{det} E}$ has become a necessary prerequisite in the study of the quantum Hamiltonian constraint. The fact that the quantum dynamics can still be made well defined, in spite of the non-polynomiality of the Hamiltonian, was first demonstrated in the context of the lattice theory [15]. A quantum Hamiltonian for the continuum 
theory was constructed in [16]. In order to get rid of the inverse powers of $\widehat{\operatorname{det} E}$ that occur naturally, an identity was employed by which the dreibeins $e_{a}^{i}$ (which are nonpolynomial functions of the inverse, densitized dreibeins $E_{i}^{a}$ that constitute half of the basic canonical variables) can be re-expressed as the Poisson brackets of $A_{a}^{i}$ with the total volume function, $e_{a}^{i}(x)=2\left\{A_{a}^{i}(x), \int d^{3} y \sqrt{\operatorname{det} E(y)}\right\}$. (This identity holds only if no modulus signs are used around the $\operatorname{det} E$-term, otherwise one has to include a factor of $\operatorname{sign}(\operatorname{det} E$ ) in the equation.) In the quantization one substitutes the Poisson brackets by commutators. Again the volume operator plays a pivotal role.

Another role of the operator $\widehat{\operatorname{det} E}$ was pointed out recently [17]. This is related to the fact that one has to impose a constraint $\operatorname{det} E>0$ classically, if the basic variables are chosen to be the Yang-Mills conjugate pairs $\left(A_{a}^{i}, E_{i}^{a}\right)$. This constraint distinguishes the phase space of gravity already at the kinematical level from that of a gauge theory. In the lattice theory, one can show that all non-vanishing eigenvalues of $\operatorname{det} E$ come in pairs of opposite sign [17], and a quantum analogue of det $E>0$ can be imposed. In practice, this requires an explicit diagonalization of the operator $\widehat{\operatorname{det} E}$.

Finally, another application is the possible inclusion of a cosmological constant term of the form $\lambda \int d^{3} x \sqrt{\operatorname{det} E(x)}$ in the Hamiltonian, with the integral taken over the entire spatial manifold. There are suggestions that this may be necessary in order to construct a non-trivial continuum limit of the (discretized) theory [18].

Our present knowledge of the spectrum of $\widehat{\operatorname{det} E}$ or the volume operator is only partial. It was observed in $[9,10]$ that its spectrum is discrete and non-zero contributions can only come from intersections of the Wilson loop states it acts on. Its diagonalization can in a so-called spin-network basis [19] be reduced to a diagonalization on finitedimensional subspaces of Hilbert space. In [7], we gave a general proof for why intersections have to be at least four-valent in order to give a non-trivial contribution. Part of the non-vanishing spectrum for such four-valent intersections was first given in [8]. These calculations were confirmed in [11], where also a general formula for the matrix elements of the volume operator was derived. These were given in terms of an orthogonal basis, obtained by decomposing spin-network states (closely related to Wilson loop states) with $n$-valent intersections into three-valent ones. Another general expression for matrix elements, with respect to a different orthogonal basis, was given in [14], together with formulae for spectra for simple special cases of classes of fourvalent intersections. (In order to avoid confusion, it should be pointed out that different volume operators may differ by overall factors and modulus signs (see, for example, the comments in [13]). Still, results on their spectra tend to be closely related.)

As we mentioned above, $\overline{\operatorname{det} E}$ can be diagonalized on finite-dimensional subspaces. These are given by fixing the flux line (or spin) assignments on the edges incoming at a given intersection where $\widehat{\operatorname{det} E}$ acts. Another way of saying this is that there exist operators associated with these edges that commute with $\widehat{\operatorname{det} E}$. On the lattice, these are of the form $\sqrt{\sum_{i} \hat{E}_{i}^{a}(l) \hat{E}^{a i}(l)}$ (no sum over $a$ ) with spectrum $\sqrt{j(j+2) / 4}, j=$ $0,1,2, \ldots$, and related to the measurement of area in the $a$-direction [20].

One does not expect to be able to derive analytic formulae for the spectrum of $\widehat{\operatorname{det} E}$ 
as the dimension of the finite-dimensional subspaces obtained by fixing the quantum numbers $j$ for the incoming edges grows. The explicit evaluation of the spectrum is therefore limited by one's computational ability to diagonalize large matrices. A suitable choice of basis in which the matrix elements are evaluated will eventually also be determined by the form of the Hamiltonian.

In this paper we construct further local operators, related to the geometry of the intersection of a spin network state, that commute with $\widehat{\operatorname{det} E}$, and lead to a further reduction of the finite-dimensional Hilbert spaces into smaller subspaces on which $\widehat{\operatorname{det} E}$ can be diagonalized separately. In mathematical terms, we will be analyzing the irreducible representations of a discrete local symmetry group of the quantum states, and decompose the Hilbert space accordingly. The method is general and can also be applied to other operators, most importantly the Hamiltonian constraint. This leads to a considerable simplification, as we will demonstrate for a class of six-valent intersections, with some of the resulting "superselection sectors" becoming totally non-degenerate with respect to their volume spectrum.

Our analysis will take place on a cubic lattice, and is therefore valid for all quantum states that can be realized on connected subsets of edges from such a lattice. The discrete symmetry group is in this case the 24-element group $\mathcal{O}$, the so-called cubic or orthogonal group [21]. The analysis may be generalized to intersections with a different geometry and symmetry group.

In the next section, we introduce a labelling of local spin network states (more precisely, a labelling for the so-called intertwiners at an intersection) with a simple transformation behaviour under the cubic group. For the construction of the superselection sectors, the relevant discrete group is a subgroup $\mathcal{O}^{(6)}$ of the cubic group times the $\mathbb{Z}_{2}$-factor associated with total spatial reflection. We describe its irreducible representations and give an explicit construction for states transforming according to a given irreducible representation from the elements of $\mathcal{O}^{(6)} \times \mathbb{Z}_{2}$ orbits. In Section 3 we give the explicit form of the operator $\widehat{\operatorname{det} E}$ associated with a vertex (or intersection) $n$. For a special class of six-valent intersections, corresponding to flux line assignments $j=(j, j, j, j, j, j)$, we perform the orbit decomposition, the construction of the superselection sectors, and diagonalization of $\widehat{\operatorname{det} E}$ up to $j=10$. All eigenvalues, together with their multiplicities in the individual sectors, are listed in Table 9. We end in Section 4 with a summary of our results and a brief discussion of the condition $\operatorname{det} E>0$.

\section{Action of the orthogonal group on the quantum state space}

Our kinematical quantum state space is the gauge-invariant sector of the Hilbert space of an $S U(2)$ lattice gauge theory in the Hamiltonian formulation [22]. Following the philosophy of spin network states [19], we use flux line labels $j=0,1,2, \ldots$ for lattice edges or links $l$. As an overcomplete set of labels for the contractors or intertwiners situated at a vertex $n$ of the lattice we use 9 numbers $j_{i k}, i, k=1,2,3$ [17]. The index $i$ denotes the incoming edges at $n$ from the 1-, 2-, or 3-direction (with respect to some 
Table 1

Multiplication table for the subgroup $\mathcal{O}^{(6)}$ of the octagonal group

\begin{tabular}{lllllll}
\hline & $\mathbf{1}$ & $\boldsymbol{R}_{1}$ & $R_{2}$ & $R_{3}$ & $S_{1}$ & $S_{2}$ \\
\hline $\mathbf{1}$ & $\mathbf{1}$ & $R_{1}$ & $R_{2}$ & $R_{3}$ & $S_{1}$ & $S_{2}$ \\
$R_{1}$ & $R_{1}$ & $\mathbf{1}$ & $S_{1}$ & $S_{2}$ & $R_{2}$ & $R_{3}$ \\
$R_{2}$ & $R_{2}$ & $S_{2}$ & $\mathbf{1}$ & $S_{1}$ & $R_{3}$ & $R_{1}$ \\
$R_{3}$ & $R_{3}$ & $S_{1}$ & $S_{2}$ & $\mathbf{1}$ & $R_{1}$ & $R_{2}$ \\
$S_{1}$ & $S_{1}$ & $R_{3}$ & $R_{1}$ & $R_{2}$ & $S_{2}$ & $\mathbf{1}$ \\
$S_{2}$ & $S_{2}$ & $R_{2}$ & $R_{3}$ & $R_{1}$ & $\mathbf{1}$ & $S_{1}$ \\
\hline
\end{tabular}

fixed orientation for the three lattice axes), and the index $k$ the corresponding outgoing edges. That is, $j_{12}$ denotes the number of (unoriented) flux lines routed through $n$ between the $(-1)$ - and the 2-direction, etc.. It is convenient to arrange the set of nine numbers into matrix form,

$$
J:=\left(\begin{array}{lll}
j_{11} & j_{12} & j_{13} \\
j_{21} & j_{22} & j_{23} \\
j_{31} & j_{32} & j_{33}
\end{array}\right) .
$$

Given flux line assignments $j_{i}, i=-1,-2,-3,1,2,3$, for the in- and outgoing links, it is easy to generate all allowed intertwiner configurations $J$. The elements of the rows and columns of $J$ simply have to add up to the appropriate $j_{i}$, for example, $\sum_{i=1}^{3} j_{1, i}=j_{-1}$, $\sum_{i=1}^{3} j_{i, 1}=j_{1}$. General elements of the cubic group $\mathcal{O}$ do not map intertwiners of the form (2.1) into themselves (but lead to configurations that by virtue of Mandelstam identities can be re-expressed as linear combinations of $J$-configurations). However, a six-dimensional subgroup $\mathcal{O}^{(6)}$ of $\mathcal{O}$ leaves the label set invariant. Apart from the identity transformation, the non-trivial elements of this subgroup $\mathcal{O}^{(6)}$ act on the $J$ 's according to

$$
\begin{array}{rll}
R_{1}(J):=\left(\begin{array}{lll}
j_{11} & j_{31} & j_{21} \\
j_{13} & j_{33} & j_{23} \\
j_{12} & j_{32} & j_{22}
\end{array}\right), & R_{2}(J):=\left(\begin{array}{lll}
j_{33} & j_{23} & j_{13} \\
j_{32} & j_{22} & j_{12} \\
j_{31} & j_{21} & j_{11}
\end{array}\right), \\
R_{3}(J):=\left(\begin{array}{lll}
j_{22} & j_{12} & j_{32} \\
j_{21} & j_{11} & j_{31} \\
j_{23} & j_{13} & j_{33}
\end{array}\right), & & \\
S_{1}(J):=\left(\begin{array}{lll}
j_{22} & j_{23} & j_{21} \\
j_{32} & j_{33} & j_{31} \\
j_{12} & j_{13} & j_{11}
\end{array}\right), & S_{2}(J):=\left(\begin{array}{lll}
j_{33} & j_{31} & j_{32} \\
j_{13} & j_{11} & j_{12} \\
j_{23} & j_{21} & j_{22}
\end{array}\right) .
\end{array}
$$

The multiplication table for the group $\mathcal{O}^{(6)}$ is given in Table 1 . We will also need the total space reflection $T$, corresponding to the transform of the matrix $J$,

$$
T(J):=\left(\begin{array}{lll}
j_{11} & j_{21} & j_{31} \\
j_{12} & j_{22} & j_{32} \\
j_{13} & j_{23} & j_{33}
\end{array}\right) .
$$


Table 2

Character table for the group $\mathcal{O}^{(6)} \times \mathbb{Z}_{2}$

\begin{tabular}{lrrrrrr}
\hline & $\{\mathbf{1}\}$ & $\left\{R_{i}\right\}$ & $\left\{S_{i}\right\}$ & $\{T\}$ & $\left\{T R_{i}\right\}$ & $\left\{T S_{i}\right\}$ \\
\hline$A_{1}^{+}$ & 1 & 1 & 1 & 1 & 1 & 1 \\
$A_{2}^{+}$ & 1 & -1 & 1 & 1 & -1 & 1 \\
$E^{+}$ & 2 & 0 & -1 & 2 & 0 & -1 \\
$A_{1}^{-}$ & 1 & 1 & 1 & -1 & -1 & -1 \\
$A_{2}^{-}$ & 1 & -1 & 1 & -1 & 1 & -1 \\
$E^{-}$ & 2 & 0 & -1 & -2 & 0 & 1 \\
\hline
\end{tabular}

Since $T$ commutes with all elements of $\mathcal{O}^{(6)}$, adjoining it we obtain a 12-element group $\mathcal{O}^{(6)} \times T \equiv \mathcal{O}^{(6)} \times \mathbb{Z}_{2}$. This group is important because it is a subgroup of the classical invariance group of the lattice function ( $\operatorname{det} E)^{2}$ (see Section 3 below). There is still a redundancy in the set of allowed $J$ 's which is associated with Mandelstam constraints and consists of all identities of the form

$$
\begin{aligned}
& \left(\begin{array}{ccc}
j_{11}+1 & j_{12} & j_{13} \\
j_{21} & j_{22}+1 & j_{23} \\
j_{31} & j_{32} & j_{33}+1
\end{array}\right)-\left(\begin{array}{ccc}
j_{11}+1 & j_{12} & j_{13} \\
j_{21} & j_{22} & j_{23}+1 \\
j_{31} & j_{32}+1 & j_{33}
\end{array}\right) \\
& -\left(\begin{array}{ccc}
j_{11} & j_{12}+1 & j_{13} \\
j_{21}+1 & j_{22} & j_{23} \\
j_{31} & j_{32} & j_{33}+1
\end{array}\right)+\left(\begin{array}{ccc}
j_{11} & j_{12}+1 & j_{13} \\
j_{21} & j_{22} & j_{23}+1 \\
j_{31}+1 & j_{32} & j_{33}
\end{array}\right) \\
& +\left(\begin{array}{cccc}
j_{11} & j_{12} & j_{13}+1 \\
j_{21}+1 & j_{22} & j_{23} \\
j_{31} & j_{32}+1 & j_{33}
\end{array}\right)-\left(\begin{array}{ccc}
j_{11} & j_{12} & j_{13}+1 \\
j_{21} & j_{22}+1 & j_{23} \\
j_{31}+1 & j_{32} & j_{33}
\end{array}\right)=0 .
\end{aligned}
$$

As already mentioned in the introduction, our aim is to identify the irreducible representations of the discrete group $\mathcal{O}^{(6)}\left(\mathcal{O}^{(6)} \times \mathbb{Z}_{2}\right)$, and construct corresponding superselection sectors on which the operator $\widehat{\operatorname{det} E}\left((\widehat{\operatorname{det} E})^{2}\right)$ can be diagonalized separately. The group $\mathcal{O}^{(6)}$ contains three conjugacy classes of elements namely, $\{\mathbf{1}\},\left\{R_{1}, R_{2}, R_{3}\right\}$ and $\left\{S_{1}, S_{2}\right\}$.

Following Ref. [21], one establishes the existence of three irreducible representations: two one-dimensional ones (called $A_{1}$ and $A_{2}$ ) and one two-dimensional one (called $E$ ). They can be identified by the values of their characters, i.e. the traces of the matrices representing the group elements (which only depend on the conjugacy class). The enlarged group $\mathcal{O}^{(6)} \times T$ has six conjugacy classes and six irreducible representations, since each of the previous representations gives rise to one of positive and one of negative parity, denoted by a subscript + or - . The characters for the group $\mathcal{O}^{(6)} \times T$ are given in Table 2.

In order to establish the contents of irreducible representations of some general representation, one can make use of the following character formula. It relates the multiplicity $m_{R}$ of a given irreducible representation $R$ in a general representation $\mathcal{M}$ to the number $n_{K}$ of elements in the conjugacy class $K$, the traces $\chi_{K}^{\mathcal{M}}$ of matrices in the representation $\mathcal{M}$ in class $K$, and the characters $\chi_{K}^{R}$ of the irreducible representation $R$, 
Table 3

Representation contents of the $\mathcal{O}^{(6)} \times \mathbb{Z}_{2}$ orbits

\begin{tabular}{ll}
\hline 1-d even & $A_{1}^{+}(1)$ \\
2-d odd & $A_{1}^{+}(1), A_{1}^{-}(1)$ \\
3-d even & $A_{1}^{+}(1), E^{+}(2)$ \\
6-d odd & $A_{1}^{+}(1), A_{1}^{-}(1), E^{+}(2), E^{-}(2)$ \\
6-d even & $A_{1}^{+}(1), A_{2}^{+}(1), E^{+}(4)$ \\
12-d odd & $A_{1}^{+}(1), A_{1}^{-}(1), A_{2}^{+}(1), A_{2}^{-}(1), E^{+}(4), E^{-}(4)$ \\
\hline
\end{tabular}

$$
m_{R}=\frac{1}{d} \sum_{K} n_{K} \chi_{K}^{\mathcal{M}} \chi_{K}^{R}
$$

For the group $\mathcal{O}^{(6)}, d=6$, and for the group $\mathcal{O}^{(6)} \times \mathbb{Z}_{2}, d=12$.

The possible orbit sizes that occur under the action of the group $\mathcal{O}^{(6)} \times \mathbb{Z}_{2}$ on states of the form (2.1) are 1, 2, 3, 6 and 12. We distinguish between parity-even and parity-odd orbits. In the former, all elements are parity-invariant, i.e. $T(J)=J$, whereas in the latter $T(J) \neq J$ for all $J$. The contents of irreducible representations of the individual orbit types is listed in Table 3 (the numbers in brackets denote the number of $J$-states).

Instead of diagonalizing a maximal set of commuting operators built from the elements of $\mathcal{O}^{(6)} \times \mathbb{Z}_{2}$ (for example, given by $\left\{1, R_{1}+R_{2}+R_{3}, S_{1}+S_{2}, T\right\}$ ) in a given finitedimensional sector of Hilbert space, we have found it computationally simpler to first construct the orbits and from those the states transforming according to a definite irreducible representation. One way of how this may be done is illustrated in Table 4 (by $J$ we denote in this context an arbitrary, fixed element of an orbit). Note that $A_{2}^{+}$-states change sign whenever one of the $R_{i}$ is applied. The prescriptions for the $E$-states in the two-dimensional representations are of course non-unique. Remember also that (2.4) induces a residual redundancy in the sets of states constructed according to Table 4, leading to relations among elements from different orbits. For the explicit calculations of the next section (which were performed using Mathematica on a DEC AlphaStation $2554 / 232$ with $64 \mathrm{Mb}$ RAM) these could be handled without particular problems.

\section{Determining the spectrum of the operator $(\operatorname{det} E)$}

We will now apply the results of the previous section to the discretized version $\operatorname{det} E(n)$ of the function (1.1), acting at a lattice vertex $n$. The corresponding selfadjoint quantum operator we will call $\hat{D}(n)$ (for convenience rescaled by a factor of $\frac{1}{6}$ with respect to the definition in [8]). In terms of the symmetrized link momenta $\hat{p}_{i}(n, \hat{a})$, it is given as

$$
\hat{D}(n):=\frac{1}{3 !} \eta_{\hat{a} \hat{b} \hat{c}} \epsilon^{i j k} \hat{p}_{i}(n, \hat{a}) \hat{p}_{j}(n, \hat{b}) \hat{p}_{k}(n, \hat{c}),
$$

where 
Table 4

How to construct states transforming in a given irreducible representation from the elements of $\mathcal{O}^{(6)} \times \mathbb{Z}_{2}$ orbits

$A_{\mathrm{I}}^{+} \quad$ all orbits: $(1+T)\left(J+R_{1} J+R_{2} J+R_{3} J+S_{1} J+S_{2} J\right)$

$A_{1}^{-} \quad$ all odd orbits: $(1-T)\left(J+R_{1} J+R_{2} J+R_{3} J+S_{1} J+S_{2} J\right)$

$A_{2}^{+} \quad$ 6-d even orbit: $J+S_{1} J+S_{2} J-R_{1} J-R_{1} S_{1} J-R_{1} S_{2} J$

12-d odd orbit: $(1+T)\left(J+S_{1} J+S_{2} J-R_{1} J-R_{1} S_{1} J-R_{1} S_{2} J\right)$

$A_{2}^{-}$12-d odd orbit: $(1+T)\left(J+S_{1} J+S_{2} J-R_{1} J-R_{1} S_{1} J-R_{1} S_{2} J\right)$

$E^{+} \quad$ 3-d even orbit: $J-S_{1} J, J-S_{2} J$, 6-d odd orbit: $(1+T)\left(J-S_{1} J\right),(1+T)\left(J-S_{2} J\right)$

6-d even orbit: $\left(1-S_{1}\right)\left(J+R_{1} J\right),\left(1-S_{2}\right)\left(J+R_{1} J\right),\left(1-S_{1}\right)\left(R_{2} J+S_{2} J\right)$,

$\left(1-S_{2}\right)\left(R_{2} J+S_{2} J\right)$

12-d odd orbit: $(1+T)\left(1-S_{1}\right)\left(J+R_{1} J\right),(1+T)\left(1-S_{2}\right)\left(J+R_{1} J\right)$,

$(1+T)\left(1-S_{1}\right)\left(R_{2} J+S_{2} J\right),(1+T)\left(1-S_{2}\right)\left(R_{2} J+S_{2} J\right)$

$E^{-} \quad$ 6-d odd orbit: $(1-T)\left(J-S_{1} J\right),(1+T)\left(J-S_{2} J\right)$

12-d odd orbit: $(1-T)\left(1-S_{1}\right)\left(J+R_{1} J\right),(1-T)\left(1-S_{2}\right)\left(J+R_{1} J\right)$,

$(1-T)\left(1-S_{1}\right)\left(R_{2} J+S_{2} J\right),(1-T)\left(1-S_{2}\right)\left(R_{2} J+S_{2} J\right)$

$$
\hat{p}_{i}(n, \hat{a})=\frac{i}{2}\left(X_{+}^{i}(n, \hat{a})+X_{-}^{i}\left(n-1_{\hat{a}}, \hat{a}\right)\right),
$$

and $X_{ \pm}^{i}(n, \hat{a})$ denote the left- and right-invariant vector fields on the group manifold associated with the link $l=(n, \hat{a})$, with commutators $\left[X_{ \pm}^{i}, X_{ \pm}^{j}\right]= \pm \epsilon^{i j k} X_{ \pm}^{k}$.

The key observation is that the classical lattice function $D(n) \equiv \operatorname{det} E(n)$ is invariant under the action of the 24-element cubic group $\mathcal{O}$ [21] (whose elements can be thought of as the orientation-preserving permutations of the three (oriented) lattice axes meeting at the intersection $n$ ). By contrast, $D(n)$ changes sign under the total space reflection $T$ (i.e. under simultaneous inversion of the three axes).

As a result of this classical symmetry, eigenstates of $\hat{D}(n)$ can be classified according to the irreducible representations of $\mathcal{O}$. This set-up is familiar to lattice gauge theorists, because it has been employed in analyzing the glueball spectrum of the Hamiltonian in four-dimensional $S U$ (3) lattice gauge theory [23]. As explained in Section 2, in the present $S U(2)$ context it is convenient to work with a set of local states that is partially gauge-fixed with respect to the $\mathcal{O}$-action, leaving us with $\mathcal{O}^{(6)}$ as the residual symmetry group.

The action of the operator $\hat{D}(n)$ on states of the form (2.1) can be obtained by considering all possible ways in which the triple derivatives contained in $\hat{D}(n)$ can act on sets of flux lines routed through the vertex $n$. Its explicit form is given by

$$
\begin{aligned}
& \hat{D}\left(\begin{array}{lll}
j_{11} & j_{12} & j_{13} \\
j_{21} & j_{22} & j_{23} \\
j_{31} & j_{32} & j_{33}
\end{array}\right) \\
& \quad=\frac{i}{4} j_{11} j_{22} j_{33}\left[\left(\begin{array}{ccc}
j_{11}-1 & j_{12}+1 & j_{13} \\
j_{21} & j_{22}-1 & j_{23}+1 \\
j_{31}+1 & j_{32} & j_{33}-1
\end{array}\right)-\left(\begin{array}{ccc}
j_{11}-1 & j_{12} & j_{13}+1 \\
j_{21}+1 & j_{22}-1 & j_{23} \\
j_{31} & j_{32}+1 & j_{33}-1
\end{array}\right)\right]
\end{aligned}
$$


$+\frac{i}{16} j_{12} j_{23} j_{31}\left[\left(\begin{array}{ccc}j_{11} & j_{12}-1 & j_{13}+1 \\ j_{21}+1 & j_{22} & j_{23}-1 \\ j_{31}-1 & j_{32}+1 & j_{33}\end{array}\right)-\left(\begin{array}{ccc}j_{11}+1 & j_{12}-1 & j_{13} \\ j_{21} & j_{22}+1 & j_{23}-1 \\ j_{31}-1 & j_{32} & j_{33}+1\end{array}\right)\right]$
$+\frac{i}{16} j_{13} j_{21} j_{32}\left[\left(\begin{array}{ccc}j_{11}+1 & j_{12} & j_{13}-1 \\ j_{21}-1 & j_{22}+1 & j_{23} \\ j_{31} & j_{32}-1 & j_{33}+1\end{array}\right)-\left(\begin{array}{ccc}j_{11} & j_{12}+1 & j_{13}-1 \\ j_{21}-1 & j_{22} & j_{23}+1 \\ j_{31}+1 & j_{32}-1 & j_{33}\end{array}\right)\right]$ $+\frac{i}{16} j_{11} j_{32}\left(j_{12}+2 j_{33}-j_{13}-j_{21}+2 j_{22}+j_{31}+2\right)\left(\begin{array}{ccc}j_{11}-1 & j_{12}+1 & j_{13} \\ j_{21} & j_{22} & j_{23} \\ j_{31}+1 & j_{32}-1 & j_{33}\end{array}\right)$ $+\frac{i}{16} j_{11} j_{23}\left(j_{12}-2 j_{22}-j_{13}-j_{21}-2 j_{33}+j_{31}-2\right)\left(\begin{array}{ccc}j_{11}-1 & j_{12} & j_{13}+1 \\ j_{21}+1 & j_{22} & j_{23}-1 \\ j_{31} & j_{32} & j_{33}\end{array}\right)$ $+\frac{i}{16} j_{22} j_{13}\left(j_{23}+2 j_{11}-j_{21}-j_{32}+2 j_{33}+j_{12}+2\right)\left(\begin{array}{ccc}j_{11} & j_{12}+1 & j_{13}-1 \\ j_{21} & j_{22}-1 & j_{23}+1 \\ j_{31} & j_{32} & j_{33}\end{array}\right)$ $+\frac{i}{16} j_{22} j_{31}\left(j_{23}-2 j_{33}-j_{21}-j_{32}-2 j_{11}+j_{12}-2\right)\left(\begin{array}{ccc}j_{11} & j_{12} & j_{13} \\ j_{21}+1 & j_{22}-1 & j_{23} \\ j_{31}-1 & j_{32}+1 & j_{33}\end{array}\right)$ $+\frac{i}{16} j_{33} j_{21}\left(j_{31}+2 j_{22}-j_{32}-j_{13}+2 j_{11}+j_{23}+2\right)\left(\begin{array}{ccc}j_{11} & j_{12} & j_{13} \\ j_{21}-1 & j_{22} & j_{23}+1 \\ j_{31}+1 & j_{32} & j_{33}-1\end{array}\right)$
$+\frac{i}{16} j_{33} j_{12}\left(j_{31}-2 j_{11}-j_{32}-j_{13}-2 j_{22}+j_{23}-2\right)\left(\begin{array}{ccc}j_{11} & j_{12}-1 & j_{13}+1 \\ j_{21} & j_{22} & j_{23} \\ j_{31} & j_{32}+1 & j_{33}-1\end{array}\right)$ $-\frac{i}{16} j_{23}\left(j_{11} j_{12}+j_{12} j_{22}+j_{12} j_{33}+j_{12} j_{13}+2 j_{12}\right)\left(\begin{array}{ccc}j_{11} & j_{12}-1 & j_{13}+1 \\ j_{21} & j_{22}+1 & j_{23}-1 \\ j_{31} & j_{32} & j_{33}\end{array}\right)$ $-\frac{i}{16} j_{31}\left(j_{22} j_{23}+j_{23} j_{33}+j_{11} j_{23}+j_{21} j_{23}+2 j_{23}\right)\left(\begin{array}{ccc}j_{11} & j_{12} & j_{13} \\ j_{21}+1 & j_{22} & j_{23}-1 \\ j_{31}-1 & j_{32} & j_{33}+1\end{array}\right)$ $-\frac{i}{16} j_{12}\left(j_{31} j_{33}+j_{11} j_{31}+j_{22} j_{31}+j_{31} j_{32}+2 j_{31}\right)\left(\begin{array}{ccc}j_{11}+1 & j_{12}-1 & j_{13} \\ j_{21} & j_{22} & j_{23} \\ j_{31}-1 & j_{32}+1 & j_{33}\end{array}\right)$ $+\frac{i}{16} j_{13}\left(j_{11} j_{21}+j_{21} j_{22}+j_{21} j_{33}+j_{21} j_{23}+2 j_{21}\right)\left(\begin{array}{ccc}j_{11}+1 & j_{12} & j_{13}-1 \\ j_{21}-1 & j_{22} & j_{23}+1 \\ j_{31} & j_{32} & j_{33}\end{array}\right)$ $+\frac{i}{16} j_{21}\left(j_{22} j_{32}+j_{32} j_{33}+j_{11} j_{32}+j_{31} j_{32}+2 j_{32}\right)\left(\begin{array}{ccc}j_{11} & j_{12} & j_{13} \\ j_{21}-1 & j_{22}+1 & j_{23} \\ j_{31}+1 & j_{32}-1 & j_{33}\end{array}\right)$ $+\frac{i}{16} j_{32}\left(j_{13} j_{33}+j_{11} j_{13}+j_{13} j_{22}+j_{12} j_{13}+2 j_{13}\right)\left(\begin{array}{ccc}j_{11} & j_{12}+1 & j_{13}-1 \\ j_{21} & j_{22} & j_{23} \\ j_{31} & j_{32}-1 & j_{33}+1\end{array}\right)$ 


$$
\begin{aligned}
& +\frac{i}{8} j_{11} j_{22}\left(j_{23}-j_{32}+j_{31}-j_{13}\right)\left(\begin{array}{ccc}
j_{11}-1 & j_{12}+1 & j_{13} \\
j_{21}+1 & j_{22}-1 & j_{23} \\
j_{31} & j_{32} & j_{33}
\end{array}\right) \\
& +\frac{i}{8} j_{11} j_{33}\left(j_{12}-j_{21}+j_{23}-j_{32}\right)\left(\begin{array}{ccc}
j_{11}-1 & j_{12} & j_{13}+1 \\
j_{21} & j_{22} & j_{23} \\
j_{31}+1 & j_{32} & j_{33}-1
\end{array}\right) \\
& +\frac{i}{8} j_{22} j_{33}\left(j_{12}-j_{21}+j_{31}-j_{13}\right)\left(\begin{array}{ccc}
j_{11} & j_{12} & j_{13} \\
j_{21} & j_{22}-1 & j_{23}+1 \\
j_{31} & j_{32}+1 & j_{33}-1
\end{array}\right) \\
& +\frac{i}{16}\left(j_{11}\left(j_{12} j_{31}-j_{12} j_{32}+j_{13} j_{23}-j_{13} j_{21}+j_{21} j_{23}-j_{31} j_{32}+j_{23}-j_{32}\right)\right. \\
& +j_{22}\left(j_{21} j_{31}-j_{12} j_{13}+j_{12} j_{23}-j_{21} j_{32}+j_{31} j_{32}-j_{13} j_{23}+j_{31}-j_{13}\right) \\
& +j_{33}\left(j_{12} j_{13}-j_{21} j_{23}+j_{23} j_{31}-j_{21} j_{31}+j_{12} j_{32}-j_{13} j_{32}+j_{12}-j_{21}\right) \\
& +j_{12} j_{13} j_{23}-j_{12} j_{13} j_{32}+j_{21} j_{23} j_{31}-j_{13} j_{21} j_{23}+j_{12} j_{31} j_{32}-j_{21} j_{31} j_{32} \\
& \left.+j_{12} j_{23}+j_{12} j_{31}-j_{13} j_{21}-j_{13} j_{32}+j_{23} j_{31}-j_{21} j_{32}\right)\left(\begin{array}{ccc}
j_{11} & j_{12} & j_{13} \\
j_{21} & j_{22} & j_{23} \\
j_{31} & j_{32} & j_{33}
\end{array}\right) .
\end{aligned}
$$

Note that the $J$-states are not orthogonal with respect to the Haar measure on the lattice. Still, we will see that within the superselection sectors of the cubic group, degeneracy of the eigenvalues of $\hat{D}(n)$ is largely lifted, which makes orthogonality largely automatic. One can verify the following conjugation relations by using the explicit form (3.3) for the operator $\hat{D}$ :

$$
\begin{aligned}
R_{i} \hat{D} R_{i} & =\hat{D}, \quad i=1,2,3, \\
S_{i} \hat{D} S_{i} & =\hat{D}, \quad i=1,2, \\
T \hat{D} T & =-\hat{D},
\end{aligned}
$$

whence it follows that $\hat{D}$ obeys the (anti-) commutation relations

$$
\begin{aligned}
{\left[\hat{D}, R_{i}\right] } & =0, \quad i=1,2,3, \\
{\left[\hat{D}, S_{1}+S_{2}\right] } & =0, \\
{[\hat{D}, T]_{+} } & =0 .
\end{aligned}
$$

We conclude that $\hat{D}$ does not alter the $\mathcal{O}^{(6)}$ quantum numbers, but maps positive- into negative-parity states and vice versa. This latter property suggests a different approach to the diagonalization of $\hat{D}(n)$. Firstly, it follows from $[\hat{D}, T]_{+}=0$ that $\left[\hat{D}^{2}, T\right]=0$. Secondly, we have shown in [17] that eigenstates of $\hat{D}$ can be obtained from those of $\hat{D}^{2}$. For any eigenstate $\chi$ of $\hat{D}^{2}$ with eigenvalue $v \neq 0, \hat{D}^{2}(n) \chi=v^{2} \chi$, one may construct a pair of eigenstates of $\hat{D}(n)$ with eigenvalues $\pm v$, namely,

$$
\hat{D}\left(\chi \pm \frac{1}{|v|} \hat{D} \chi\right)= \pm \frac{1}{|v|} \hat{D}^{2} \chi+\hat{D} \chi= \pm|v|\left(\chi \pm \frac{1}{|v|} \hat{D} \chi\right) \text {. }
$$

Since in our search for eigenstates we would like to reduce the size of matrices that have to be diagonalized, it is simpler to diagonalize $\hat{D}(n)^{2}$ first, and then construct 
Table 5

Numbers of $\mathcal{O}^{(6)} \times \mathbb{Z}_{2}$ orbits for states with $j=(j, j, j, j, j, j)$

\begin{tabular}{lllllrrrrrr}
\hline$j$ & 1 & 2 & 3 & 4 & 5 & 6 & 7 & 8 & 9 & 10 \\
\hline 1-d even & 1 & 2 & 2 & 3 & 3 & 4 & 4 & 5 & 5 & 6 \\
2-d odd & 1 & 2 & 4 & 6 & 9 & 12 & 16 & 20 & 25 & 30 \\
3-d even & 1 & 3 & 5 & 9 & 12 & 18 & 22 & 30 & 35 & 45 \\
6-d odd & 0 & 1 & 4 & 9 & 18 & 30 & 48 & 70 & 100 & 135 \\
6-d even & 0 & 0 & 1 & 2 & 5 & 8 & 14 & 20 & 30 & 40 \\
12-d odd & 0 & 0 & 0 & 1 & 3 & 8 & 16 & 30 & 50 & 80 \\
total & 3 & 8 & 16 & 30 & 50 & 80 & 120 & 175 & 245 & 336 \\
\hline
\end{tabular}

eigenstates of $\hat{D}(n)$ using (3.6). The explicit form for $\hat{D}(n)^{2}$ contains 142 terms and can be obtained from (3.3).

One further observation turns out to be useful. Since parity-odd wave functions are constructed by weighted sums (with factors \pm 1 ) of spin network states, which may sometimes vanish, there are always fewer states transforming according to the representations $A_{i}^{-}, E^{-}$, than those transforming according to $A_{i}^{+}, E^{+}$. The most efficient way of diagonalizing $\hat{D}$ (and the one which we follow below) is therefore to start from the wave functions transforming in one of the negative-parity irreducible representations, diagonalize $\hat{D}^{2}$, construct the images under $\hat{D}$ of the resulting states (which all have positive parity), and then form linear combinations according to (3.6). The number of zero-volume states is given by the difference of positive- and negative-parity states.

As an application of the scheme outlined above, we have analyzed the irreducible representation contents of the sub-Hilbert spaces with flux line numbers

$$
\boldsymbol{j}=\left(j_{-1}, j_{-2}, j_{-3}, j_{1}, j_{2}, j_{3}\right)=(j, j, j, j, j, j)
$$

(i.e. for genuine six-valent intersections) up to $j=10$, and obtained the spectrum of $\hat{D}(n)$. This class of configurations is special in the sense that states with fixed $j$ are mapped into themselves by the action of $\mathcal{O}^{(6)} \times \mathbb{Z}_{2}$. The numbers of orbits of a given type for fixed $j$ (before imposing the constraints (2.4)) are listed in Table 5.

We then proceeded as described above by diagonalizing $\hat{D}(n)^{2}$ separately on the superselection sectors corresponding to different quantum numbers for the action of $\mathcal{O}^{(6)} \times \mathbb{Z}_{2}$. The resulting numbers of linearly independent eigenstates of $\hat{D}(n)$ with strictly positive and vanishing eigenvalues are listed in Tables 6 and 7.

Comparing the rows for the $A_{1^{-}}, A_{2}$ - and $E$-sectors in Table 6 to the total numbers of states in the Hilbert spaces before the $\mathcal{O}^{(6)} \times T$-action is taken into account (Table 8 ), one observes that the matrix sizes are reduced considerably when the superselection sectors are treated separately. Another interesting feature is that the number of zeroeigenvalues grows less rapidly with $j$ than that of the non-vanishing ones (by curve fits we found the dependences $\frac{3}{4} j^{2}+\frac{3}{2} j+\frac{3}{4}$ for $j$ odd and $\frac{3}{4} j^{2}+\frac{3}{2} j+1$ for $j$ even, as opposed to $\left.\frac{1}{2} j^{3}+\frac{3}{2} j^{2}+2 j+1\right)$.

Finally, here are our results for the non-negative eigenvalues of the operator $\hat{D}(n)=$ $\widehat{\operatorname{det} E(n)}$. The numbers in Table 9 are given in length units, i.e. we have taken the sixth 
Table 6

Numbers of linearly independent eigenstates of $\hat{D}(n)$ with eigenvalue $>0$

\begin{tabular}{lrrrrrrrrrr}
\hline$j$ & 1 & 2 & 3 & 4 & 5 & 6 & 7 & 8 & 9 & 10 \\
\hline$A_{1}$ & 1 & 2 & 5 & 8 & 14 & 20 & 30 & 40 & 55 & 70 \\
$A_{2}$ & 0 & 0 & 0 & 1 & 2 & 5 & 8 & 14 & 20 & 30 \\
$E$ & 0 & 2 & 6 & 14 & 26 & 44 & 68 & 100 & 140 & 190 \\
total & 1 & 4 & 11 & 23 & 42 & 69 & 106 & 154 & 215 & 290 \\
\hline
\end{tabular}

Table 7

Numbers of linearly independent eigenstates of $\hat{D}(n)$ with eigenvalue $=0$

\begin{tabular}{lllllllllll}
$j$ & 1 & 2 & 3 & 4 & 5 & 6 & 7 & 8 & 9 & 10 \\
\hline total & 3 & 7 & 12 & 19 & 27 & 37 & 48 & 61 & 75 & 91 \\
\hline
\end{tabular}

Table 8

Number of linearly independent states with fixed flux lines $j=(j, j, j, j, j, j)$

\begin{tabular}{ccccccccccc}
$j$ & 1 & 2 & 3 & 4 & 5 & 6 & 7 & 8 & 9 & 10 \\
\hline total & 5 & 15 & 34 & 65 & 111 & 175 & 260 & 369 & 505 & 671 \\
\hline
\end{tabular}

root of the eigenvalues of $\hat{D}(n)$. The three numbers in brackets give the degeneracy of eigenvalues in the $A_{1^{-}}, A_{2^{-}}$and $E$-sectors, respectively.

The spectrum is rather complex, and becomes more and more spread out with increasing $j$. There seem to be only three different degeneracy patterns, $(1,0,0),(1,0,2)$ and $(1,1,4)$. Every eigenvalue that occurs is already contained in the $\mathcal{O}^{(6)}$-invariant (i.e. the $A_{1^{-}}$) sector, and is non-degenerate. It would be interesting to see whether this is also the case for more general flux line configurations. Note also that the highest eigenvalue for fixed $j$ is always non-degenerate. The "volume deficit" observed in [24] (the fact that all volume eigenvalues are systematically smaller than expected from the (Laplacian) area eigenvalues, compared to the relation one would obtain for a Euclidean reference metric), persists for higher $j$, although it becomes less pronounced.

\section{Summary and outlook}

We have explained in this paper how the diagonalization of the volume operator in the loop representation of quantum gravity can be simplified by taking into account discrete symmetries at the intersections of the Wilson loop states. Applied to the case of up to six-valent intersections on a cubic lattice, this requires the decomposition of Hilbert space into the irreducible representations of a six-dimensional subgroup $\mathcal{O}^{(6)}$ of the cubic group. One finds a set of local operators that commute with $\widehat{\operatorname{det} E}$, and therefore can be diagonalized simultaneously. A further simplification arises when one includes the total spatial reflection, and diagonalizes the operator $(\widehat{\operatorname{det} E})^{2}$, from which the eigenstates 
Table 9

Values for $x^{1 / 6}$, where $x$ is a non-negative eigenvalue of $\hat{D}(n)$

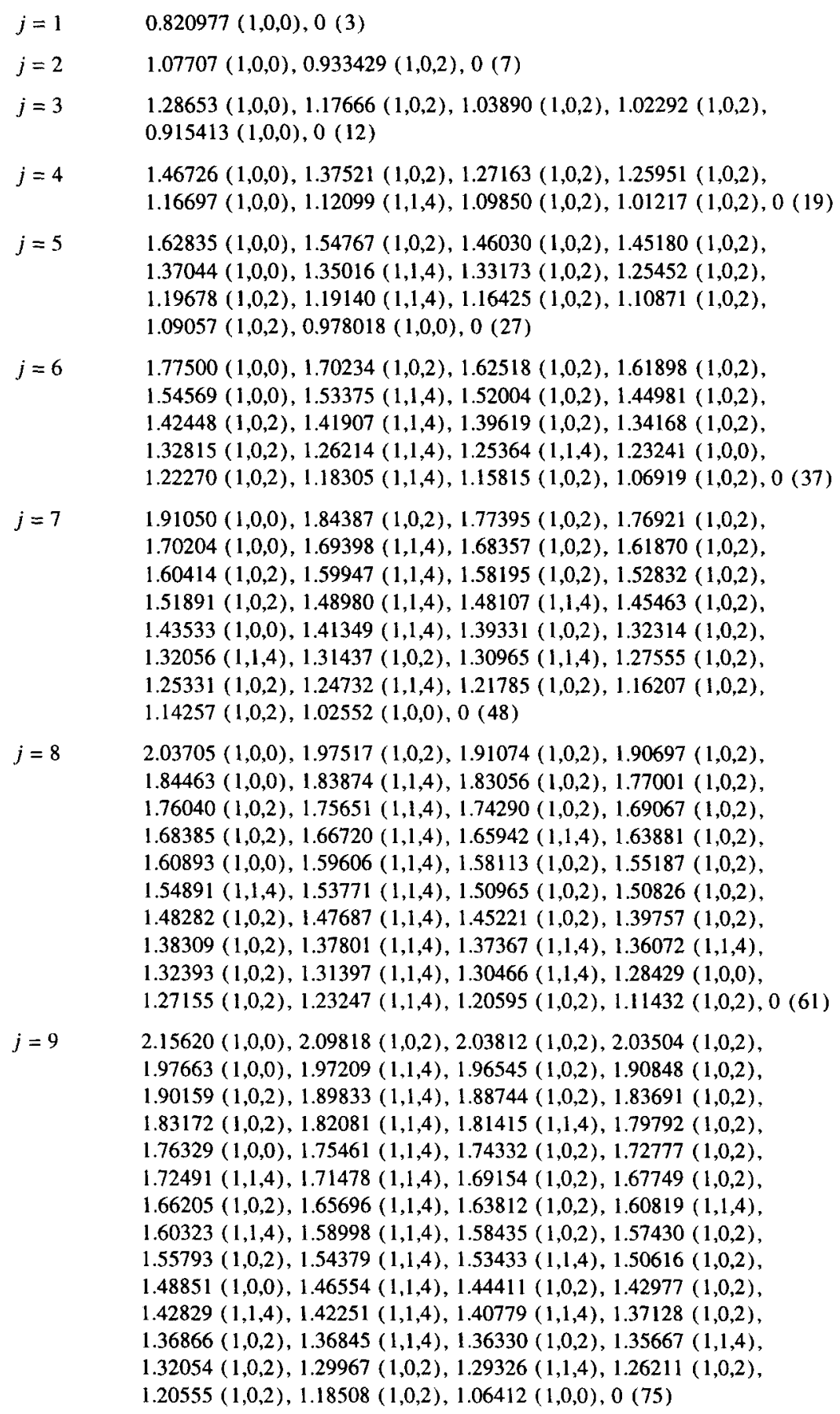


Table 9 -continued

$$
\begin{array}{rl}
j=10 & 2.26912(1,0,0), 2.21432(1,0,2), 2.15784(1,0,2), 2.15526(1,0,2), \\
& 2.10012(1,0,0), 2.09649(1,1,4), 2.09097(1,0,2), 2.03703(1,0,2), \\
& 2.03180(1,0,2), 2.02905(1,1,4), 2.02009(1,0,2), 1.97130(1,0,2), \\
& 1.96720(1,0,2), 1.95944(1,1,4), 1.95376(1,1,4), 1.94061(1,0,2), \\
& 1.90385(1,0,0), 1.89752(1,1,4), 1.88867(1,0,2), 1.87925(1,0,2), \\
& 1.87664(1,1,4), 1.86783(1,1,4), 1.84934(1,0,2), 1.82734(1,0,2), \\
& 1.81716(1,0,2), 1.81294(1,1,4), 1.79834(1,0,2), 1.78328(1,1,4), \\
& 1.77840(1,1,4), 1.76633(1,1,4), 1.74596(1,0,2), 1.74079(1,0,2), \\
& 1.73868(1,0,2), 1.72121(1,1,4), 1.71284(1,1,4), 1.69092(1,0,2), \\
& 1.66208(1,0,0), 1.66200(1,0,2), 1.66017(1,1,4), 1.65364(1,1,4), \\
& 1.64853(1,1,4), 1.63864(1,1,4), 1.63269(1,0,2), 1.60428(1,0,2), \\
& 1.60238(1,0,2), 1.59918(1,1,4), 1.58717(1,1,4), 1.55981(1,0,2), \\
& 1.55607(1,0,2), 1.53183(1,0,2), 1.52550(1,1,4), 1.49950(1,0,2), \\
& 1.47744(1,1,4), 1.47486(1,1,4), 1.46780(1,1,4), 1.45154(1,1,4), \\
& 1.44430(1,0,2), 1.42912(1,0,2), 1.42294(1,1,4), 1.41825(1,1,4), \\
& 1.41035(1,0,2), 1.40442(1,1,4), 1.36572(1,0,2), 1.35747(1,1,4), \\
& 1.34758(1,1,4), 1.32752(1,0,0), 1.31283(1,0,2), 1.27374(1,1,4), \\
& 1.24600(1,0,2), 1.15189(1,0,2), 0(91)
\end{array}
$$

of $\widehat{\operatorname{det} E}$ can be obtained using the results in [17]. To demonstrate the viability of the method, we have calculated the spectrum for a class of six-valent intersections with flux line numbers $(j, j, j, j, j, j)$, for $j \leqslant 10$. We found that all eigenvalues are already contained in the $\mathcal{O}^{(6)}$-invariant sector, without degeneracy. It is conceivable that this sector is also distinguished on physical grounds, but this depends on how the continuum limit of the lattice theory will be taken.

Let us close with some comments on the condition $\widehat{\operatorname{det} E}>0$ which, as we have argued in [17], should be imposed on the quantum state space. It remains to be seen at which stage of the quantization it is imposed most conveniently. Ignoring for the moment the zero-eigenvalue states, this condition reduces the dimensionality of the Hilbert space by a factor $2^{x}$, where $x$ is the number of intersections of the lattice. In addition, one obtains a condition on physical operators $\hat{\mathcal{P}}$, namely, that they should not map out of the subspace of states with $\widehat{\operatorname{det} E}$ eigenvalues $\geqslant 0$ (or $>0$ ). A sufficient condition is given by the vanishing of the commutator

$$
[\hat{\mathcal{P}}, \operatorname{sign}(\widehat{\operatorname{det} E})]=0 .
$$

However, we have not found a simple form of this condition which would not require the explicit knowledge of the eigenstates of $\widehat{\operatorname{det} E}$. Note that (4.1) is a rather strong condition which, for example, is not satisfied by the lattice analogues of the area operators defined in [25]. A less stringent condition is to require that (4.1) be satisfied in the limit as the lattice spacing is taken to zero. This is also sensible from a physical point of view, since the condition should be independent of the regularization (for example, the version of the area operator corresponding to a pure Laplacian $[10,20]$ obviously fulfills (4.1), but coincides with other discretized forms of the area to lowest order in the lattice spacing).

We do not know whether there is a way to formulate a condition like $\widehat{\operatorname{det} E}>0$ in the 
continuum theory. The regularization used for the volume operator in [14] forces one to use modulus signs around the operator, in order that the square root $\sqrt{|\widehat{\operatorname{det} E}|}$ is welldefined, something not necessary in the case of the lattice regularization. Nevertheless, we think that such a constraint on states, along with operator conditions of the type (4.1), should be imposed in the quantum theory - even if this leads to new complications because they describe a property of the gravitational theory.

\section{References}

[1] A. Ashtekar, New variables for classical and quantum gravity, Phys. Rev. Lett. 57 (1986) 2244; A new Hamiltonian formulation of general relativity, Phys. Rev. D 36 (1987) 1587.

[2] R.S. Tate, Polynomial constraints for general relativity using real geometrodynamical variables, Class. Quant. Grav. 9 (1992) 101.

[3] C. Rovelli and L. Smolin, Loop space representation of quantum general relativity, Nucl. Phys. B 331 (1990) 80.

[4] Articles by B. Brügmann, R. Loll, T. Thiemann and A. Ashtekar, in Canonical gravity: from classical to quantum, ed. J. Ehlers and H. Friedrich (Springer, Berlin, 1994);

R. Loll, Still on the way to quantizing gravity, to appear in Proc. 12th Italian Conf. on General Relativity and Gravitational Physics, Rome, Italy, 23-27 September 1996.

[5] P. Renteln and L. Smolin, A lattice approach to spinorial quantum gravity, Class. Quant. Grav. 6 ( 1989 ) 275;

P. Renteln, Some results of SU(2) spinorial lattice gravity, Class. Quant. Grav. 7 (1990) 493;

O. Boström, M. Miller and L. Smolin, A new discretization of classical and quantum general relativity, Syracuse U. preprint SU-GP-93-4-1;

R. Loll, Non-perturbative solutions for lattice quantum gravity, Nucl. Phys. B 444 (1995) 619;

K. Ezawa, Multi-plaquette solutions for discretized Ashtekar gravity, Mod. Phys. Lett. A 11 (1996) 2921;

H. Fort, R. Gambini and J. Pullin, Lattice knot theory and quantum gravity in the loop representation, to appear in Phys. Rev. D.

[6] J.F. Barbero G., Real Ashtekar variables for Lorentzian signature space-times, Phys. Rev. D 51 (1995) 5507.

[7] R. Loll, The volume operator in discretized quantum gravity, Phys. Rev, Lett. 75 (1995) 3048.

[8] R. Loll, Spectrum of the volume operator in quantum gravity, Nucl. Phys. B 460 (1996) 143.

[9] L. Smolin, Recent developments in non-perturbative quantum gravity, in Quantum gravity and cosmology (World Scientific, Singapore, 1992) p. 3.

[10] C. Rovelli and L. Smolin, Discreteness of area and volume in quantum gravity, Nucl. Phys. B 442 (1995) 593; B 456 (1995) 753 (E).

[11] R. De Pietri and C. Rovelli, Geometry eigenvalues and scalar product from recoupling theory in loop quantum gravity, Phys. Rev. D 54 (1996) 2664.

[12] A. Ashtekar and J. Lewandowski, Differential geometry on the spaces of connections via graphs and projective limits, J. Geom. Phys. 17 (1995) 191.

[13] J. Lewandowski, Volume and quantizations, Class. Quant. Grav. 15 (1997) 71.

[14] T. Thiemann, Closed formula for the matrix elements of the volume operator in canonical quantum gravity, Harvard U. preprint HUTMP-96/B-353.

[15] R. Loll, A real alternative to quantum gravity in loop space, Phys. Rev. D 54 (1996) 5381.

[16] T. Thiemann, Anomaly free formulation of non-perturbative four-dimensional Lorentzian quantum gravity, Phys. Lett. B 380 (1996) 257; Quantum spin dynamics I \& II, Harvard U. preprints HUTMP96/B-351 and B-352.

[17] R. Loll, Imposing det $E>0$ in discrete quantum gravity, Phys. Lett. B 399 (1997) 227.

[18] L. Smolin, Instability and absence of long-ranged correlations in non-perturbative quantum general relativity, Penn State U. preprint CGPG-96/9-4. 
[19] C. Rovelli and L. Smolin, Spin networks and quantum gravity, Phys. Rev. D 52 (1995) 5743;

J.B. Baez, Spin network states in gauge theory, Adv. Math. 117 (1996) 253; Spin networks and non-perturbative quantum gravity, in The interface of knots and physics, ed. L. Kauffman, American Mathernatical Society, Providence, 1996;

L. Smolin, The future of spin networks, Penn State U. preprint CGPG-97.

[20] R. Loll, Further results on geometric operators in quantum gravity, Class. Quant. Grav. 14 (1997) 1725.

[21] T. Janssen, Crystallographic groups (North-Holland, Amsterdam, 1973);

S.L. Altmann, in Quantum Theory II, ed. D.R. Bates (Academic Press, New York, 1962).

[22] J. Kogut and L. Susskind, Hamiltonian formulation of Wilson's lattice gauge theories, Phys. Rev. D 11 (1975) 395;

J.B. Kogut, The lattice gauge theory approach to quantum chromodynamics, Rev. Mod. Phys. 55 (1983) 775 .

[23] B. Berg and A. Billoire, Glueball spectroscopy in 4d SU(3) lattice gauge theory(I), Nucl. Phys. B 221 (1983) 109.

[24] R. Loll, Latticing quantum gravity, Nucl. Phys. B (Proc. Suppl.) 57 (1997) 255.

[25] A. Ashtekar and J. Lewandowski, Quantum theory of geometry I: area operators, Class. Quant. Grav. 14 ( 1997) A55. 